\title{
Relato de experiência do projeto de extensão universitária remota: Readaptações - Um olhar resiliente em meio a pandemia
}

\author{
Online community extension project's experience report: Readaptations - A resilient look through \\ the pandemic \\ Informe de experiencia del proyecto de extensión universitaria A Distancia: Readaptaciones - Una \\ mirada resiliente en medio de la pandemia
}

Thalia Santos Silva

ORCID: https://orcid.org/0000-0001-8247-5437 Centro Universitário Cesmac, Brasil

E-mail: th.s.silva@hotmail.com

Camila Holanda Cavalcante Matos

ORCID: https://orcid.org/0000-0001-8283-0590 Centro Universitário Cesmac, Brasil

E-mail: camilaacavalvantem@outlook.com

Marina de Omena Souza Costa ORCID: https://orcid.org/0000-0002-7426-4047 Centro Universitário Cesmac, Brasil

E-mail: marinaomenacosta@gmail.com

Renata Cordeiro de Godoy Miranda ORCID: https://orcid.org/0000-0002-7224-4173

Centro Universitário Cesmac, Brasil

E-mail: renatagodoymiranda@ hotmail.com

Arthur Ravel Teles de Lima Bezerra ORCID: https://orcid.org/0000-0001-7096-7899 Centro Universitário Cesmac, Brasil E-mail: arthurravel@hotmail.com

Camila Maria de Lima Santos ORCID: https://orcid.org/0000-0002-0061-1472 Centro Universitário Cesmac, Brasil E-mail: camilima472@gmail.com

Gabriela Maria Calixto Barros Sampaio Fernandes ORCID: https://orcid.org/0000-0003-3614-9555 Centro Universitário Cesmac, Brasil

E-mail: gabrielacalixtobsf@gmail.com João de Deus Santos Neto ORCID: https://orcid.org/0000-0001-8442-0085 Centro Universitário Cesmac, Brasil E-mail: jd.sn68@gmail.com

Manuela Chagas Nascimento da Silva ORCID: https://orcid.org/0000-0001-9340-5936 Centro Universitário Cesmac, Brasil E-mail: manucachagas@hotmail.com

Maria Eduarda Lima Moraes Sarmento ORCID: https://orcid.org/0000-0003-2886-4911 Centro Universitário Cesmac, Brasil eduarda.sarmento@outlook.com.br

Angela Líbia Chagas Amaral ORCID: https://orcid.org/0000-0002-1479-2067 Centro Universitário Cesmac E-mail: angelalibia@ hotmail.com Naianne Ramos Monteiro ORCID: https://orcid.org/0000-0003-0479-4306 Centro Universitário Cesmac, Brasil E-mail: naianneramos@ hotmail.com

\section{Resumo}

A criação desse projeto de extensão fundamentou-se na ideia de contribuir para minimizar questões em atual carência no âmbito social e de saúde, provocadas pela pandemia do COVID-19, além de complementar, de maneira estratégica 
com conteúdo do programa do curso de Odontologia. Com o objetivo de gerar informações e conhecimento para população em geral, envolver a comunidade acadêmica e externa na difusão e debate das temáticas apresentadas sobre saúde e odontologia; o desenvolvimento das temáticas foi realizado através da produção de lives, vídeos e postagens na plataforma Instagram ${ }^{\circledR}$. O material produzido através das redes sociais proporciona o rápido compartilhamento de informações, curiosidades e conhecimentos sobre a saúde bucal em conjunto com a saúde em geral. As métricas obtidas demonstram o grande alcance das postagens, assim como abrange a cobertura das atividades extensionistas, com a possibilidade de ser realizada de maneira remota. Após a conclusão do projeto, foi possível perceber o grande alcance das redes sociais, o impacto que a área da saúde pode causar na população - de forma positiva -, por meio de compartilhamento de conhecimentos e informações, a fim de proporcionar melhor qualidade de vida e saúde.

Palavras-chave: Saúde bucal; Atenção Primária à Saúde; Relações comunidade- instituição; Pandemias; Rede social; Ensino.

\begin{abstract}
The creation of this Online Community Extension Project is based on the idea of minimizing current socio-economic and health deficiency issues, caused by the COVID-19 pandemic, while strategically complementing with existing Odontology content. In order to spread information and knowledge to the population in general, involve the academic and non-academic community on debating and disseminating the themes in the project about health and dentistry; the development of these subjects was made through videos, lives and posts on Instagram's ${ }^{\circledR}$ platform. The produced content through social media enables fast sharing of information, knowledge and curiosities about oral health along with sistemic health. The obtained results show the high spread of the posts, as well as including extensionists projects, which has the possibility of being made online. After this project's conclusion, it was possible to notice the great social media range, the positive impact that the healthcare field can cause on population through sharing knowledge and information, in order to provide better life and health quality.
\end{abstract}

Keywords: Oral health; Primary Health Care; Community-institutional relations; Pandemics; Social networking; Teaching.

\title{
Resumen
}

La creación de este proyecto de extensión se basó en la idea de contribuir a minimizar los problemas de necesidad actual en el ámbito social y de la salud, provocados por la pandemia COVID-19, además de complementar estratégicamente el contenido del programa del curso de Odontología. Con el fin de generar información y conocimiento para la población en general, involucrar a la comunidad académica y externa en la difusión y debate de los temas presentados sobre salud y odontología; el desarrollo de los temas se llevó a cabo a través de la producción de videos en vivo, videos y publicaciones en la plataforma Instagram ${ }^{\circledR}$. El material elaborado a través de las redes sociales permite compartir rápidamente informaciones, curiosidades y conocimientos sobre la salud bucal en conjunto con la salud en general. Las métricas obtenidas demuestran el amplio alcance de los puestos, así como la cobertura de las actividades de extensión, con posibilidad de realizarse de forma remota. Al finalizar el proyecto, se pudo ver el amplio alcance de las redes sociales, el impacto que el área de salud puede tener en la población, de manera positiva, a través del intercambio de conocimientos e información, con el fin de brindar una mejor calidad de vida y salud.

Palabras clave: Salud bucal; Atención Primaria de Salud; Relaciones comunidad-institución; Pandemias; Red social; Ensenãnza.

\section{Introdução}

O final de dezembro de 2019 foi marcado pela emergência de uma pandemia, tendo posteriormente sua causa identificada como o novo Betacoronavírus, também chamado COVID-19, que exigiu uma readaptação no cotidiano da sociedade. Devido à sua rápida propagação causou um enorme impacto social, econômico e sanitário, sendo um dos maiores desafios em escala mundial do ponto de vista sanitário (Moutinho, 2020).

A COVID-19 é uma doença que afeta pessoas de várias maneiras, apresentando sintomas variados de acordo com cada indivíduo, podendo se apresentar de forma assintomática; sintomas mais comuns e leves, como febre e tosse seca; a quadros respiratórios graves, levando ao óbito. Entretanto em adultos, idosos e pessoas acometidas por comorbidades como diabetes, obesidade, asma, doença cardíaca ou pulmonar e hipertensão, podem ter mais riscos de complicações de seu quadro clínico (Pereira et al, 2020; Sohrabi et al., 2020).

Com a crescente disseminação do vírus, os governantes decidiram coibir aglomerações de pessoas, fechamento de comércios e serviços considerados não essenciais, fechamento de universidades e escolas, como também locais que pudessem gerar concentração de pessoas. Assim, diante do impacto ocasionado, a Lei Federal $\mathrm{n}^{\circ} 13.979$, de 6 de fevereiro de 2020 
estabeleceu o isolamento social como medida para reduzir a propagação do vírus e enfrentamento da emergência de saúde pública (EUFRÁSIO, 2020). Também foram adotadas outras medidas para contenção da disseminação como o isolamento de pessoas infectadas e quarentena para suspeitos da infecção, recomendação da lavagem frequente das mãos, uso de máscaras e álcool à 70\% (Ministério Da Saúde, 2020; FrateschI et al., 2020).

Com a nova medida de isolamento social adotada, estabelecimentos comerciais não essenciais tiveram que ser fechados temporariamente, assim como as unidades de ensino, as escolas e universidade de todo o território nacional (Pereira et al., 2020). Visando a continuidade das atividades acadêmicas nas instituições de ensino, em 17 de março de 2020, o governo brasileiro regulamentou uma portaria (Portaria $\mathrm{N}^{\circ} 343$ ), autorizando a substituição de aulas presenciais em meios digitais enquanto durar a situação da pandemia, a fim de manter as atividades dos cursos de graduação. (Beahr, 2020; Wu e McGoogan, 2020)

A propagação de incansáveis informações falsas (fake news) nas redes sociais e informações contraditórias sobre o distanciamento social corroboram para impactos negativos no enfretamento da pandemia, além de aumentar o nível de preocupação das pessoas diante do problema mundial (Matos, 2020).

Com o intuito de manter os alunos engajados em atividades acadêmicas durante esse período de pandemia, as universidades precisaram reinventar-se, dando continuidade às atividades curriculares e extracurriculares de modo remoto. Para minimizar questões em atual carência no âmbito social e contribuir para a formação dos discentes e docentes na integração com a comunidade, a extensão universitária, por sua vez, precisou aderir ao ensino remoto.

Sendo a extensão universitária definida como um processo educativo, cultural e científico que agrega pesquisa e ensino de forma integrada, possibilitando uma relação de mudança proveitosa e benéfica entre universidade e sociedade. (Carneiro, 2011; Carneiro, 2015)

Posto isso, a realização desse projeto fundamentou-se na ideia de disseminar informações contempladas no programa do curso de odontologia e saúde em geral de forma remota, articulando a prática do conhecimento científico do ensino e da pesquisa com as necessidades da comunidade.

O planejamento e execução das atividades do projeto: Readaptações - Um olhar resiliente em meio a pandemia, promovem a remodelação da formação acadêmica, de modo que docentes e discentes se posicionam frente a um obstáculo enfrentado pela comunidade e disponibilizam sua atuação para solucionar ou minimizar essa carência. O público-alvo tornou-se multiplicador das ações, além de desenvolver uma responsabilidade social comunitária, ressaltando a importância da integração, serviço, ensino e comunidade.

\section{Metodologia}

Este estudo, trata-se de um relato de experiência, caracterizado como um estudo descritivo, de abordagem qualitativa. Sendo o relato de experiência um meio da pesquisa descritiva que evidência uma ação, retratando uma situação vivenciada no ambiente profissional de interesse da comunidade científica (Flick, 2013).

Relata-se a realização e a excussão de uma extensão universitária, de forma remota, durante o período de isolamento social ocasionado pela Pandemia do Coronavírus. Formada por acadêmicos e discentes de Odontologia, de uma Instituição de Nível Superior do estado de Alagoas. Foi criado um perfil em uma rede social e diariamente postado diversas temáticas relacionadas à saúde geral e saúde bucal.

\section{Relato de Experiência}

O projeto de extensão intitulado: Readaptações - Um olhar resiliente em meio a pandemia, fez parte do edital de extensão comunitária remota voluntária nº 01/2020, do Centro Universitário Cesmac (Maceió-AL). 
A equipe de extensão foi formada por dez discentes do curso de odontologia e duas docentes, essas responsáveis pela revisão e correções das postagens antes de sua publicação. Inicialmente foram realizadas capacitações com os discentes, através da plataforma online de comunicação disponível pela instituição, com o intuito de calibrar os extensionistas para que os tornassem qualificados e cientes sobre as temáticas que seriam tratadas ao longo da extensão.

Para execução do projeto, foi criada uma conta vinculada a rede social Instagram ${ }^{\circledR}$ onde seriam publicadas as postagens elaboradas pelo projeto e em seguida propagada para a comunidade profissional, acadêmica e geral; um grupo de WhatsApp ${ }^{\circledR}$ e na plataforma de ensino da Instituição, onde ambos eram usados como meio de comunicação entre extensionistas e coordenadoras.

O perfil na plataforma Instagram ${ }^{\circledR}$ disponibiliza diversos dados a respeito do alcance das publicações, informando métricas como: quantidade de curtidas, comentários, encaminhamentos, salvamentos, engajamento, contas alcançadas, interação com o conteúdo, número de seguidores adquiridos, dia e horário de melhor engajamento do público alvo, visitas ao perfil, entre outras informações.

As publicações através do Instagram ${ }^{\circledR}$ seguiam um tema semanal, relacionado aos acontecimentos do momento ou informações relevantes à população. Todas as postagens seguiam o seguinte planejamento: a) Criação e sugestão do tema pela dupla de extensionistas da semana; b) Discussão e ajustes do tema com a coordenação da extensão; c) Elaboração das artes e legendas; d) Apreciação e discussão entre os demais extensionistas; e) Aprovação final das postagens; f) Divulgação das artes ao longo da semana.

De acordo com os dados fornecidos pelo Instragram ${ }^{\circledR}$ e perfil dos seguidores, foi usada uma estratégia de melhor horário de publicação para maior interação com a comunidade.

Ao longo do período de atividade, foram tratadas de temáticas como: saúde geral, saúde oral, saúde mental e odontologia. Todos os temas foram pensados de forma a gerar uma conexão e relação de confiança com o público. Os posts eram realizados através de fotos, confecção de vídeos postados via IGTV, stories e Reels no aplicativo Instagram ${ }^{\circledR}$ buscando sempre utilizar uma linguagem simples, atual, que tornassem as publicações mais atraentes e consequentemente, com maior alcance da informação. Algumas semanas foram realizadas lives, com convidados relacionados ao tema proposto.

A conta pode ser encontrada através do usuário @ readaptcesmac, que até os dias atuais se encontra ativa.

\section{Discussão}

Os projetos de extensão universitária fazem parte das funções sociais das instituições de ensino, com o fito de viabilizar o acesso à informação a sociedade, assim como promover o desenvolvimento dos mesmos e fomentar projetos. A extensão é essencial para o fortalecimento e articulação do conhecimento científico com a necessidade da comunidade, interagindo e transformando a realidade social (Eufrasio, 2020).

A extensão universitária é de suma importância na vida do discente e do público em geral, pois, o indivíduo beneficiado usufrui do aprendizado, provocando assim, mudanças sociais. Outrossim, essa modalidade de ensino é fundamental no processo de formação pessoal, acadêmica e profissional, haja vista que pode exercer e efetivar o compromisso com a qualidade de vida da população, com ações sócio-educativas (Rodrigues, 2013)

Tendo em vista o aspecto da pandemia do COVID-19, o crescimento exponencial dos meios digitais influenciou de forma positiva para a continuidade do ensino remoto, bem como das estratégias de promoção de saúde e interação entre a universidade e comunidade, assim, cumprindo a função social da universidade. Esse contexto foi de suma importância, devido à incerteza de quanto tempo seria necessário para o retorno das atividades presenciais (Oliveira, 2019; Eufrasio, 2020). 
Levando em consideração a situação do Brasil e do mundo, frente a pandemia do COVID-19, surgiu um novo ambiente que demonstrou a necessidade de execução de atividades remotas, tanto para difusão de informações confiáveis, como para aproximar a relação entre docentes e discentes na elaboração dessas atividades.

Os discentes do curso de odontologia do Centro Universitário Cesmac (Maceió-AL) tiveram a oportunidade de inscrição e participação em projetos de extensão comunitária remota voluntária, para proporcionar atividades extensionistas extracurriculares, a fim de favorecer também a saúde mental, possibilitando a vivência de atividades lúdicas, culturais, laborais e das diferentes áreas de conhecimento para as comunidades acadêmica.

As atividades executadas tiveram a finalidade de contribuir ao desenvolvimento de ações que estabeleçam troca de saberes, conhecimentos e experiências, alicerçando como um processo educativo integrados à realidade social (Rodrigues, 2013). As ações foram realizadas na plataforma Instagram, para compartilhamento de informações, diminuindo barreiras entre a informação segura, embasada em fontes científicas e a população em geral.

Nesse sentido, o projeto de extensão Readaptações: um olhar resiliente em meio a pandemia foi realizado com o papel de pesquisar, simplificar e disseminar informações relacionadas a odontologia e saúde em geral de forma remota, recriando conteúdo de forma que alcançassem a comunidade, docentes e estudantes da graduação. Através de pesquisas e observando como se comporta a sociedade, foram criados os conteúdos, que eram postados de diferentes e criativas formas, avançando no sentido de construir e levar serviços de qualidade prestados a comunidade ainda que de maneira remota.

A extensão atuou no período de junho até dezembro de 2020 e encerrou suas atividades com o alcance de 3.973 seguidores no Instagram. As postagens foram desenvolvidas ao longo de 20 semanas de atuação; totalizando 75 postagens, 16 temas centrais trabalhados, 12 lives, vídeos e reels e 69.783 visualizações, podendo ser observado um grande alcance nos números de curtidas, comentários, posts salvos e compartilhamentos.

Rosa (2020), mostram que houve um sucesso nos resultados obtidos em atividades assíncronas não obrigatórias, através de um alto número de inscritos e participação ativa de grande parte dos alunos. Atrelado a isso, o estudo de Eufrasio (2020), mostra que o ensino remoto contribuiu de forma eficiente na formação dos discentes envolvidos nas atividades extracurriculares.

Por outro lado, Silva (2020) afirma que durante a pandemia do novo COVID-19, os alunos sofreram uma redução da qualidade de vida, haja vista que os mesmos vêm vivenciando uma realidade nunca enfrentada. Assim a distância física tornou o cotidiano mais solitário (Gualharde et al.,2020). Assim, sendo imprescindível a adoção de atividades realizadas no ensino a distância, como uma importante ferramenta de benfeitoria para esta realidade (Rosa et al, 2021).

Na pesquisa de Peloso (2020) a maioria dos discentes acreditam que as atividades a distância são consistentes com o conteúdo ministrado, ajudam a consolidar e melhorar o aprendizado e a frequência de ensino é adequada, embora temem que o conhecimento do material clínico e formação profissional sejam prejudicas.

Assim sendo, é evidente que a vivência do acadêmico junto a programas de ensino é, portanto, instrumento valioso para a transformação do futuro Cirurgião-dentista em profissional de saúde, pois a extensão universitária auxilia na formação técnicocientífica e, principalmente, humanista. Outrossim, proporciona ao discente que desenvolva também habilidades de comunicação, assim, promovendo a formação de profissionais mais capacitados para o mercado de trabalho (Pereira et al, 2011).

\section{Conclusão}

A partir da confecção e execução desse projeto de extensão remota, foi possível analisar a importância da classe docente e discente da área de saúde, na qualidade de vida e bem-estar da população, demonstrando que a atenção em odontologia vai além de dentes e da cavidade oral, buscando manter o a disposição física e mental do cidadão, principalmente na atual situação de pandemia, que exigiu renovações e readaptações no cotidiano mundial. Ademais, pode-se compartilhar informações e atualidades da odontologia e ainda sobre áreas da saúde geral, como a conscientização contra o câncer de mama e o câncer de 
próstata. Além da interação com a comunidade através das redes sociais, a execução de um projeto de extensão remota, proporcionou uma maior interação entre docentes e discentes, discussão de temas variados e agregação de conhecimentos além dos conteúdos contemplados na graduação. Entender que a formação profissional se estende a formação cidadã e ao comprometimento com a comunidade a qual estamos inseridos.

O grupo de extensionista tem pretensão de dar continuidade ao projeto de extensão, fazendo seleção de novos membros da instituição, renovando o projeto anualmente, a cada novo edital de extensão da instituição. Dando oportunidade para os discentes do curso de Odontologia.

\section{Referências}

Behar, P. A. (2020). O ensino remoto emergencial e a educação à distância. Jornal da Universidade. 14(08).

Carneiro, J. A., Costa, F. M. D., Lima, C. C., Otaviano, M. R., \& Fróes, G. J. (2011). Unimontes solidária: interação comunitária e prática médica com a extensão. Revista Brasileira de Educação Médica, 35(2), 283-288.

Carneiro, J. A., Da Costa, F. M., Poswar, F. D. O., \& de Freitas, M. O. S. (2014). Liga acadêmica: instrumento de ensino, pesquisa e extensão universitária. Gestão e Saúde, 6(1), pag-667.

da Equipe, C. C. D. E., \& do HC Criança, T. A prática de Mindfulness em profissionais de saúde em tempos de COVID-19: uma revisão sistemática.

Da Rosa, W. L. D. O., da Silva, A. F., Immich, F., de Araujo, T. S., Valente, L. L., \& Madruga, F. C. (2021). Experiência De Ensino Remoto Em Projeto De Extensão De Odontologia Restauradora Em Tempos De Pandemia. Expressa Extensão, 26(1), 215-226.

de Matos, R. C. (2020). Fake news frente a pandemia de COVID-19. Vigilância Sanitária em Debate: Sociedade, Ciência \& Tecnologia (Health Surveillance under Debate: Society, Science \& Technology)-Visa em Debate, 8(3), 78-85.

Dias, M. S. D. L., Brognoli, P. C., de Figueiredo Hamm, L. R., \& da Silva Neto, P. M. (2020). Extensão universitária em tempos de COVID-19: um relato de experiência no projeto (tutor). Revista Extensão \& Sociedade, 12(1).

Eufrásio, L. S., de Oliveira Anunciação, J., Silva, T. A. A., \& Magalhães, A. G. (2020). Estratégia De Telemonitoramento Para Extensão Universitária. Revista Extensão \& Sociedade, 12(1).

Flick, U. Introdução à metodologia de pesquisa: um guia para iniciantes. M. Lopes (Trad.) Penso, 2013.

Galhardi, C. P., Freire, N. P., Minayo, M. C. D. S., \& Fagundes, M. C. M. (2020). Fato ou Fake? Uma análise da desinformação frente à pandemia da Covid-19 no Brasil. Ciência \& Saúde Coletiva, 25, 4201-4210.

Lorandi, S., da Silva Loss, G. M., Malta, S. T., Graeff Filho, V. L., dos Santos, V. A., \& Iserhard, C. A. (2021). " Insetos, E Daí?”: Ressignificando As Dimensões Da Extensão Universitária Com A Pandemia Da COVID-19. Expressa Extensão, 26(1), 285-299.

Ministério da Saúde. (2020). Medidas não farmacológicas. Ministério da saúde.

Moutinho, F. F. B. (2021). Extensão Universitária: Uma Luz Na Escuridão Da Pandemia De COVID-19. Intermedius-Revista de Extensão da UNIFIMES, 1(1), $63-72$.

Nunes, R. K. S., dos Santos Maciel, G. A., Almeida, E. B., Guedes, M. R., \& Henn, R. (2021). Desafios E Adaptações Da Extensão Universitária Em Tempos De Pandemia: Relato De Experiência. Revista Ciência Plural, 7(1), 211-223.

Oliveira, E. E. D. (2019). Um auto relato sobre a participação no projeto ateliê do sorriso: um caminho percorrido da extensão à sociedade. Rev. Ciênc. Plur, 72 88 .

Peloso, R. M., Ferruzzi, F., Mori, A. A., Camacho, D. P., Franzin, L. C. D. S., Margioto Teston, A. P., \& Freitas, K. M. S. (2020). Notes from the field: concerns of health-related higher education students in Brazil pertaining to distance learning during the coronavirus pandemic. Evaluation \& the Health Professions, 43(3), 201-203.

Pereira, I., Corbo, A. D. A., Paula, T. S. G. D., Mendonça, F. C. R., \& Valle, S. (2020). Manual sobre biossegurança para reabertura de escolas no contexto da COVID-19.

Pereira, S. M., Mialhe, F. L., Pereira, L. J., de Fátima Soares, M., da Silva Tagliaferro, E. P., de Castro Meneghim, M., \& Pereira, A. C. (2011). Extensão universitária e trabalho voluntário na formação do acadêmico em Odontologia. Arquivos em Odontologia, 47(2).

Rodrigues, A. L. L., do Amaral Costa, C. L. N., Prata, M. S., Batalha, T. B. S., \& Neto, I. D. F. P. (2013). Contribuições da extensão universitária na sociedade. Caderno de Graduação-Ciências Humanas e Sociais-UNIT-SERGIPE, 1(2), 141-148.

Silva, P. G. D. B., de Oliveira, C. A. L., Borges, M. M. F., Moreira, D. M., Alencar, P. N. B., Avelar, R. L., \& Sousa, F. B. (2021). Distance learning during social seclusion by COVID-19: improving the quality of life of undergraduate dentistry students. European Journal of Dental Education, 25(1), $124-134$.

Sohrabi, C., Alsafi, Z., O'Neill, N., Khan, M., Kerwan, A., Al-Jabir, A., \& Agha, R. (2020). World Health Organization declares global emergency: A review of the 2019 novel coronavirus (COVID-19). International journal of surgery, 76, 71-76.

Wu, Z., \& McGoogan, J. M. (2020). Characteristics of and important lessons from the coronavirus disease 2019 (COVID-19) outbreak in China: summary of a report of 72314 cases from the Chinese Center for Disease Control and Prevention. Jama, 323(13), 1239-1242. 